\title{
Practice patterns of ophthalmologists administering intravitreal injections in Europe: a longitudinal survey
}

This article was published in the following Dove Press journal:

Clinical Ophthalmology

12 December 2016

Number of times this article has been viewed

\author{
Kui Huang' \\ Marla B Sultan² \\ Duo Zhou ${ }^{3}$ \\ Charles S Tressler ${ }^{4}$ \\ Jingping Mo'
}

'Epidemiology, Worldwide Safety and Regulatory, ${ }^{2}$ Clinical Sciences, Global Innovative Products Business, ${ }^{3} \mathrm{Clinical}$ Statistics, Global Established Pharma Business, ${ }^{4}$ Safety Surveillance and Risk Management, Worldwide Safety and Regulatory, Pfizer Inc, New York, NY, USA
Correspondence: Kui Huang Epidemiology, Worldwide Safety and Regulatory, Pfizer Inc, 235 East 42nd Street, New York, NY 10017, USA

Tel +l 2I2 733 I309

Fax + I 2128573672

Email kui.a.huang@pfizer.com
Purpose: This study was performed to understand the practice patterns of ophthalmologists administering intravitreal (IVT) injections in Europe after the procedure became routine.

Methods: As part of a prospective, multinational, non-interventional cohort study in 13 countries in Europe between 2006 and 2012, ophthalmologists completed the Baseline Questionnaire and the Follow-up Questionnaire 1 year after baseline.

Results and discussion: Of the 125 ophthalmologists who participated in the study, 113 (90.4\%) completed the Baseline Questionnaire. Most of these ophthalmologists were medical retina specialists (43.0\%). The median number of IVT injections that the ophthalmologists performed per month during the year prior to completing the Baseline Questionnaire was 20.0. The majority of the ophthalmologists had performed their last IVT injection prior to completing the questionnaire in an operating room or theater $(68.4 \%)$. When performing IVT injections, a majority of the ophthalmologists reported applying povidone-iodine (90.4\%) before IVT injections and topical antibiotics right after IVT injections (89.5\%). In addition, $81.6 \%$ of the ophthalmologists reported using a sterile adhesive eye drape and $80.7 \%$ reported using an eyelid speculum. In all, 95 ophthalmologists (76\%) completed the Follow-up Questionnaire. The median number of IVT injections performed per month during the year prior to completing the Follow-up Questionnaire by these ophthalmologists was increased to 35. The results of the Follow-up Questionnaire on administering IVT injections were similar to those of the Baseline Questionnaire. A majority of the ophthalmologists reported applying povidone-iodine (87.4\%) before IVT injections, topical antibiotics right after IVT injections (89.5\%), and an eyelid speculum (85.3\%).

Conclusion: The results of this study indicated a good adherence to all aspects of the guidelines on IVT injections. It seemed that ophthalmologists were more experienced in IVT injections after they became a routine treatment procedure.

Keywords: intravitreal injection, questionnaire, povidone-iodine, topical antibiotics, eyelid speculum

\section{Introduction}

Intravitreal (IVT) injections have historically been used in the treatment of endophthalmitis and pneumatic retinopexy and for administration of off-label pharmacologic agents. ${ }^{1}$ Prior to 2006, this procedure was not a routine practice in Europe.

The first vascular endothelial growth factor (VEGF) inhibitor, Macugen ${ }^{\circledR}$ (Pfizer Inc, New York, NY, USA; pegaptanib sodium), was approved for use in the treatment of neovascular age-related macular degeneration (AMD) in Europe in 2005, followed by Lucentis ${ }^{\circledR}$ (Novartis International AG, Basal, Switzerland; ranibizumab) in 2007 and Eylea (Regeneron, Tarrytown, USA; aflibercept) in 2012. Avastin (Novartis 
International AG; bevacizumab) has been used off-label for treatment of this disease since the middle of 2006. All of these agents are administered by IVT injections.

At the time of approval, given that the IVT injection was not routinely performed in Europe, educational materials aimed at safe and effective use of Macugen were distributed to ophthalmologists. The education materials consisted of two guidance articles ${ }^{2,3}$ and an explanatory CD-ROM slide presentation of the proper technique for IVT injections. One of the two articles was a review article on minimizing the risk of endophthalmitis following IVT injection ${ }^{2}$ and the other was guidelines on IVT injections. ${ }^{3}$ The guidelines were based on round-table deliberations conducted after a review of published and unpublished studies and case series on IVT injections. Under the guidelines, the use of gloves, draping, pre-injection topical antibiotics, povidone-iodine, eyelid speculum, and post-injection topical antibiotics were recommended when performing IVT injections. However, there was only one study that evaluated practice patterns of ophthalmologists administering IVT injections in Europe. ${ }^{4}$ The study was conducted in a single country in 2004.

A non-interventional study was conducted primarily to estimate the incidence of pertinent ocular adverse events (POAEs), including endophthalmitis, retinal detachment, vitreous hemorrhage, retinal tear, traumatic cataract, and increased intraocular pressure (IOP), as related to IVT injection of AMD treatments in a "real-world" setting in Europe from August 2006 to February 2012. Since no studies evaluated the practice patterns of ophthalmologists administering IVT injections across Europe, two questionnaires as part of this study were administered to better understand the practice patterns of treating ophthalmologists performing IVT injections in the study, after the introduction of the use of IVT injection as a routine treatment procedure. This article describes the practice patterns of the ophthalmologists administering IVT injections in the study.

\section{Methods}

Two ophthalmologist questionnaires were part of a 2-year, prospective, multinational, observational, cohort study in 13 countries including Belgium, Cyprus, the Czech Republic, Denmark, France, Germany, Greece, Ireland, Italy, Poland, Slovakia, Spain, and Sweden in Europe. Details of the study design are described elsewhere. ${ }^{5}$ Briefly, the primary objective of the study was to estimate the incidence of POAEs among neovascular AMD patients receiving IVT injections for AMD treatment in the real-world setting in Europe. The study was initiated in August 2006 and ended in February 2012.
Ophthalmologists in the study completed the Baseline Questionnaire and the Follow-up Questionnaire at 1 year after baseline. Data elements of both questionnaires included average number of IVT injections performed per year and detailed information on the procedure used to perform IVT injections (eg, gloves, technique, locations). The ophthalmologist questionnaire at baseline also included years ophthalmologists had been practicing in the field of ophthalmology, ophthalmologists' subspecialties, the number of IVT injections performed each month in the past year, and the number of years IVT injections were performed at this rate. Descriptive statistics were used to summarize characteristics and practice patterns related to IVT injections among ophthalmologists in the study. Ophthalmologists completed the questionnaires between 2006 and 2012, with a large majority of them completing both questionnaires between 2007 and 2011. Participant agreement/ consent was implied by completion or return of the questionnaires. This study was initially approved by an ethics committe at Sønderborg Hospital in Denmark, followed by the other ethics committees at the rest of participating sites.

\section{Results}

A total of 125 treating ophthalmologists from 66 sites in 13 countries participated in the main study. A majority of the ophthalmologists were from Slovakia, the Czech Republic, Germany, Italy, France, and Spain. Of the 125 treating ophthalmologists, 113 (90.4\%) ophthalmologists completed the Baseline Questionnaire. On average, ophthalmologists had 16 years of experience practicing in the field of ophthalmology (SD: 8.7 years). A large number of these ophthalmologists had 11-15 years of experience (21.9\%). In terms of subspecialties, most of the ophthalmologists were medical retina specialists $(43.0 \%)$, followed by surgical retina specialists $(22.8 \%)$.

Table 1 summarizes main results of the Baseline Questionnaire and Follow-up Questionnaire. According to the Baseline Questionnaire, the median number of IVT injections that the ophthalmologists performed per month during the past year was 20, with a range from 0 to 120 injections. Most of the ophthalmologists had performed 11-20 IVT injections per month during the past year (28.1\%). A large number of ophthalmologists had been performing IVT injections at the above rate for 1,2 , and 3 years $(30.7 \%, 27.2 \%$, and $14.9 \%$, respectively). A majority of the ophthalmologists had performed their last IVT injections in an operating room or a theater (68.4\%). Very few ophthalmologists performed their last IVT injections in an eye examination room (3.5\%).

When administering IVT injections, a large majority of the ophthalmologists reported having applied povidone-iodine 
Table I Results of Baseline Questionnaire and Follow-up Questionnaire

\begin{tabular}{|c|c|c|}
\hline Variables & $\begin{array}{l}\text { Baseline } \\
(n=1 \mid 3)\end{array}$ & $\begin{array}{l}\text { Follow-up } \\
(n=95)\end{array}$ \\
\hline \multicolumn{3}{|c|}{ Number of IVT injections performed per month in the past year, $n$ (\%) } \\
\hline $0-10$ & $31(27.4)$ & $17(14.9)$ \\
\hline $\mathrm{II}-20$ & $32(28.3)$ & $24(2 I .1)$ \\
\hline $21-30$ & $13(1 \mid .5)$ & $20(17.5)$ \\
\hline$>30$ & $30(26.6)$ & $51(44.8)$ \\
\hline Missing & $7(6.2)$ & $2(1.8)$ \\
\hline \multicolumn{3}{|c|}{ When giving IVT injections, the following was applied, $\mathrm{n}(\%)$} \\
\hline Povidone-iodine & $103(91.2)$ & $83(87.4)$ \\
\hline Topical antibiotics right before injection & $76(67.3)$ & $61(64.2)$ \\
\hline Sterile adhesive eye drape & $93(82.3)$ & $75(79.0)$ \\
\hline Eyelid speculum & $92(81.4)$ & $81(85.3)$ \\
\hline Topical antibiotics after injection & $102(90.3)$ & $85(89.5)$ \\
\hline \multicolumn{3}{|l|}{ Type of gloves used, n (\%) } \\
\hline Latex & $86(76.1)$ & $69(60.5)$ \\
\hline Latex free & $19(16.8)$ & $22(19.3)$ \\
\hline Data missing & $8(7.1)$ & $23(20.2)$ \\
\hline
\end{tabular}

Abbreviation: IVT, intravitreal.

(90.4\%) and topical antibiotics before IVT injections (66.7\%) and topical antibiotics right after IVT injections (89.5\%). In addition, $81.6 \%$ of the ophthalmologists reported having used a sterile adhesive eye drape and $80.7 \%$ reported having utilized an eyelid speculum. A majority of the ophthalmologists also reported the use of latex gloves (75.4\%).

Of the 125 ophthalmologists, 95 (76.0\%) completed the Follow-up Questionnaire. Based on the Follow-up Questionnaire, the median number of IVT injections performed per month by these ophthalmologists was increased to 35 with a range of 0-95 injections. The results on performing IVT injections were similar to those of the Baseline Questionnaire. A majority of the ophthalmologists reported having applied povidone-iodine $(87.4 \%)$ and topical antibiotics $(64.2 \%)$ before IVT injections, topical antibiotics right after IVT injections (89.5\%), as well as an eyelid speculum (85.3\%) and a sterile adhesive eye drape (79\%). A total of $60.5 \%$ of the ophthalmologists also reported having used latex gloves when administering IVT injections; it was noticed that $20.2 \%$ of the ophthalmologists did not respond to this question.

\section{Discussion}

There has been only one published study that described the practice patterns of ophthalmologists administering IVT injections in Europe. Prior to IVT injections becoming a routine procedure, Anijeet et al ${ }^{4}$ conducted a cross-sectional survey among ophthalmologists in the UK in 2004 ( $n=139)$. Like our study, the majority of ophthalmologists in their study were medical retina specialists or retina surgeons, who performed IVT injections in an operating theater and applied povidone-iodine before the IVT injection. But the percentage of ophthalmologists who used eye drapes and the percentage of ophthalmologists who applied topical antibiotics right after IVT injections in their study were much lower than those in our study ( $63 \%$ vs $79 \%$ and $62 \%$ vs $89.5 \%$, respectively). As expected, a vast majority of ophthalmologists in their study performed IVT injections less frequently (five to ten IVT injections per month) compared to those in this study (20-35 injections per month) because their study was conducted before the procedure became a routine practice.

Two other published studies reported practice patterns of ophthalmologists administering IVT injections in the US and Canada. Green-Simms et $\mathrm{al}^{6}$ conducted a large crosssectional survey among retinal specialists in the US in 2010 $(n=765)$. The results of our study were similar to those of their study with respect to the use of eyelid speculum, povidoneiodine before the IVT injection, and topical antibiotics after the IVT injection. A Canadian survey was conducted among retina specialists in $2012(\mathrm{n}=75) .^{7}$ Like our study, a majority of ophthalmologists in the Canadian survey used eyelid speculums. Unlike our study, however, the Canadian survey reported that a majority of retina specialists in the US and Canadian surveys did not use eye drapes and topical antibiotics before the injection; the survey also indicated that most IVT injections were performed in the office in the US and Canada. The authors speculate that differences in practice patterns reported in these studies may be due to differences in training as well as in health care systems.

As part of a non-interventional study to evaluate the incidence of POAEs involving 13 countries in Europe, two questionnaires were administered to better understand the practice patterns of treating ophthalmologists administering IVT injections in this study. The findings of this study showed that a majority of treating ophthalmologists reported use of gloves, draping, pre-injection topical antibiotics, povidoneiodine, eyelid speculum, and post-injection topical antibiotics when performing IVT injections as recommended by the guidelines. ${ }^{3}$ Thus, it was concluded that treating ophthalmologists had a good adherence to all aspects of the guidelines on IVT injections.

The findings of the main study demonstrated that the incidence of POAEs was low overall. ${ }^{5}$ Endophthalmitis was not reported in the study. The authors consider that perhaps the finding of a lower incidence of IVT injectionrelated POAEs in the main study may be attributed to a good adherence to the guidelines on IVT injections as well as the increased experiences of treating ophthalmologists during the 
study period (the number of IVT injections performed each month in the past year was reported to be increased from the Baseline Questionnaire to the Follow-up Questionnaire).

It is important to note that educational materials and two published articles on treatment guidelines of IVT injections ${ }^{2,3}$ were distributed at the investigator meetings prior to patient enrollment. Although the authors would like to draw a direct conclusion between the introduction of educational materials and the adherence to the guidelines, as noted, the questionnaires only assess knowledge rather than actual behavior; the authors consider the introduction of educational materials may provide reinforcement of knowledge. It is important to introduce guidelines and a plan to support key information on a procedure in order to minimize serious consequences associated with the procedure before it becomes routine. This is particularly important in this situation, given the recent expansion of the use of the VEGF inhibitors for not just the treatment of neovascular AMD but for other retinal diseases, including proliferative diabetic retinopathy and macular edema from a variety of disorders. Ophthalmologists should maintain vigilant and meticulous attention to the principles of the IVT injection procedure.

\section{Conclusion}

The results of this study indicated a good adherence to all aspects of the guidelines on IVT injections in Europe. It seemed that ophthalmologists were more experienced in performing IVT injections after they became a routine treatment procedure.

\section{Acknowledgments}

The authors gratefully thank patients who agreed to participate in this study and investigators of this study. This paper was presented at the 30th International Conference on Pharmacoepidemiology and Therapeutic Risk Management, October 24-27, 2014, Taipei, Taiwan, as an abstract presentation. The presentation's abstract was published in Pharmacoepidemiology and Drug Safety, Special Issue: Abstracts of the 30th International Conference on Pharmacoepidemiology and Therapeutic Risk Management, Volume 23, Issue S1, pages $1-497$, October $2014 .^{8}$ This study was supported by Pfizer Inc.

\section{Author contributions}

$\mathrm{KH}$ and JM were involved in conception and design of the study; $\mathrm{KH}$ in data collection; DZ in statistical expertise; $\mathrm{KH}$, MBS, and CST in literature search. All authors were involved in writing the manuscript; analysis and interpretation of data; critical revision of the manuscript; gave final approval for the version to be published, and agree to be accountable for all aspects of the work.

\section{Disclosure}

All authors are employees of Pfizer Inc. The authors report no other conflicts of interest in this work.

\section{References}

1. Jager RD, Mieler WF, Miller JW. Age-related macular degeneration. N Engl J Med. 2008;358(24):2606-2617.

2. Ta CN. Minimizing the risk of endophthalmitis following intravitreous injections. Retina. 2004;24(5):699-705.

3. Aiello LP, Brucker AJ, Chang S, et al. Evolving guidelines for intravitreous injections. Retina. 2004;24(5 supp1):S3-S19.

4. Anijeet DR, Hanson RJ, Bhagey J, Bates RA. National survey of the technique of intravitreal triamcinolone injection in the United Kingdom. Eye. 2007;21(4):480-486.

5. Huang K, Sultan MB, Zhou D, et al. Ocular safety of intravitreal injections of age-related macular degeneration treatments in a prospective observational cohort study in Europe. J Clin Res Ophthalmol. 2015; $3(1): 1-5$.

6. Green-Simms AE, Ekdawi NS, Bakri SJ. Survey of intravitreal injection techniques among retinal specialists in the United States. Am J Ophthalmol. 2011;151(2):329-332.

7. Xing L, Dorrepaal SJ, Gale J. Survey of intravitreal injection techniques and treatment protocols among retina specialists in Canada. Can J Ophthalmol. 2014;49(3):261-266.

8. Huang K, Sultan MB, Zhou D, Tressler CS, Mo J. Special Issue: Abstracts of the 30th International Conference on Pharmacoepidemiology and Therapeutic Risk Management, Taipei, Taiwan, October 24-27, 2014. Pharmacoepidemiol Drug Saf. 2014;23(S1):1-497.
Clinical Ophthalmology

\section{Publish your work in this journal}

Clinical Ophthalmology is an international, peer-reviewed journal covering all subspecialties within ophthalmology. Key topics include: Optometry; Visual science; Pharmacology and drug therapy in eye diseases; Basic Sciences; Primary and Secondary eye care; Patient Safety and Quality of Care Improvements. This journal is indexed on
PubMed Central and CAS, and is the official journal of The Society of Clinical Ophthalmology (SCO). The manuscript management system is completely online and includes a very quick and fair peer-review system, which is all easy to use. Visit http://www.dovepress.com/ testimonials.php to read real quotes from published authors. 